\title{
Population structure of the squid Doryteuthis (Loligo) pealeii on the eastern coast of the USA: Reply to Gerlach et al. (2012)
}

\author{
Paul W. Shaw ${ }^{1,3, *}$, Lisa Hendrickson ${ }^{2}$, Niall J. McKeown ${ }^{1}$, Terry Stonier ${ }^{3}$, \\ Marie-Jose Naud ${ }^{4}$, Warwick H. H. Sauer ${ }^{3}$ \\ ${ }^{1}$ Institute of Biological, Environmental and Rural Sciences (IBERS), Aberystwyth University, Aberystwyth SY23 3FG, UK \\ ${ }^{2}$ US National Marine Fisheries Service, Northeast Fisheries Science Center, 166 Water St., Woods Hole, Massachusetts 02543, USA \\ ${ }^{3}$ Department of Ichthyology \& Fisheries Science, Rhodes University, Grahamstown 6140, South Africa \\ ${ }^{4}$ Marine Biological Association of the UK, Citadel Hill, Plymouth, Devon PL1 2PB, UK
}

\begin{abstract}
Gerlach et al. (2012; Mar Ecol Prog Ser 450:281-283) argue that temporal instability in patterns of cryptic genetic differentiation in Doryteuthis pealeii, resulting from sampling of family groups, is the most likely explanation for differences in patterns of genetic differentiation presented in Buresch et al. (2006) and Shaw et al. (2010). We agree that temporal genetic patchiness might be an explanation, but disagree that cohesive and genetically differentiated family groups are the cause. We conclude that currently there is not enough evidence for temporally stable population differentiation, explained by a feasible biological/physical mechanism, to support management of the species as anything other than single genetic stocks across large geographical regions of the NW Atlantic. Further study of this species is warranted to understand the factors generating temporal genetic changes.
\end{abstract}

KEY WORDS: Squid $\cdot$ Population genetics $\cdot$ Fisheries management

\section{Introduction}

Buresch et al. (2006) reported substantial and significant differences in microsatellite DNA allele frequencies among Doryteuthis (then Loligo) pealeii samples collected along the eastern coast of the USA, and suggested that this indicated an underlying mechanism of subpopulation recognition by individual squid, either through high-fidelity homing to natal spawning sites or lifelong recognition and schooling with their own genetic subpopulation. In contrast, Shaw et al. (2010) reported no significant microsatellite allele frequency differences among $D$. pealeii samples collected from spawning aggregations in the same geographical area, concluding that there was no association of spawning aggregations with distinct genetic subpopulations.

Gerlach et al. (2012) reanalysed and reassessed the data presented in Buresch et al. (2006) to address points raised in Shaw et al. (2010) regarding differences in sample sizes, methods of null allele adjustment, and the possibility of temporal instability of allele frequencies.

\section{Sample sizes}

The probabilities of Type I and II errors were estimated for the range of sample sizes used in the respective data sets (Buresch et al. 2006, Shaw et al. 
2010) using the simulation method implemented in POWSIM (Ryman \& Palm 2006). This demonstrated a considerably reduced Type II (i.e. false negative) error rate for the pairwise comparisons in Shaw et al. (2010), compared to Buresch et al. (2006), with similarly low Type I (i.e. false positive) error rates for both studies.

\section{Null allele adjustment}

It is reassuring that the reanalysis conducted by Gerlach et al. (2012) indicates that the dataset adjustment applied by MICROCHECKER did not bias their original results unduly. In Shaw et al. (2010), we simply emphasized for any potential users of MICROCHECKER that the non-global genotype adjustment suggested by this programme can result in spurious elevation of $F_{\mathrm{ST}}$ values under particular conditions (loci with few, common alleles) that are met in the loci used to screen Doryteuthis pealeii.

\section{Temporal instability}

As sample sizes and data adjustment are discounted by Gerlach et al. (2012), the only source of discrepancy between the datasets is change in patterns of genetic differentiation across years. Gerlach et al. (2012) refer to unpublished data showing instability of allele frequencies between temporal samples from Woods Hole (MA, USA) to support temporal instability. They suggest that the initial spatial genetic differentiation and temporal changes result from temporal fluctuation in dispersal and migration patterns among cohesive and genetically differentiated groups of squid that remain together throughout their life, and that the apparent genetic homogeneity observed by Shaw et al. (2010) might result from storms mixing these genetically differentiated groups. To test for the presence of genetically differentiated sub-populations mixed together within the samples used in Shaw et al. (2010), we analysed the dataset using STRUCTURE (Pritchard et al. 2000). Both the 'no admixture model' (as recommended for low $F_{\mathrm{ST}}$ by Pritchard et al. 2000) and 'admixture model with correlated allele frequencies' indicated no support for mixed or recently interbreeding subpopulations (highest posterior probability for $\mathrm{K}=1$, MCMC runs with burn in of $10^{-6}$ steps followed by $5 \times 10^{-6}$ steps, 3 replicates). We conclude that mixing of differentiated family groups is an unlikely explanation of the differences between the studies. Storms are a common occurrence every year within the study area, and the yearly differences in larval dispersal patterns and adult migration routes hypothesized in Gerlach et al. (2012) have not been documented for this species: time series of spring and fall catches of Doryteuthis pealeii collected within the study area show consistent seasonal migration patterns for adults and juveniles across years (Black et al. 1987). Also, as noted in Buresch et al. (2006), there is no evidence for a mechanism by which a highly migratory annual species with non-overlapping generations such as $D$. pealeii could form and maintain cohesive genetic groups that persist throughout the life cycle. Genetic patchiness in migratory marine fish because of homing to natal spawning grounds has been reported (e.g. Ruzzante et al. 1998, Knutsen et al. 2003), but this mechanism is rejected for $D$. pealeii by both Gerlach et al. (2012) and Shaw et al. (2010).

Neither Buresch et al. (2006) nor Shaw et al. (2010) demonstrate consistent spatial and temporal genetic differentiation in Doryteuthis pealeii that can be related to definable groups of individuals. Therefore, on the basis of these data, it is impossible to define usable management units within the sampled area of the NE Atlantic.

\section{Conclusions}

The suggestion of geographically-based genetic patchiness in a populous, widespread and migratory squid species is an interesting proposition, that - if confirmed - could have intriguing possibilities for our concepts of dispersal and gene flow in mobile marine species. The pattern of differentiation described for Doryteuthis pealeii by Buresch et al. (2006) is, to date, unique amongst squid species (see references in Shaw et al. 2010), and not supported by the data in Shaw et al. (2010) or predictions from the species' life history and biology. In our opinion, the available evidence does not support local, genetically differentiated units due to spawning site philopatry or lifelong association among kin groups. We agree that it is not clear why there is a discrepancy between the 2 datasets, and we encourage further study of this species to clarify whether transitory genetic patchiness does occur. From a fisheries management perspective, until unambiguous and temporally stable genetic population structuring can be linked to features of the biology or distribution of the species, we recommend that the $D$. pealeii population located within the study area is viewed as a single genetic stock. 


\section{LITERATURE CITED}

Black GAF, Rowell TW, Dawe EG (1987) Atlas of the biology and distribution of the squids Illex illecebrosus and Loligo pealei in the Northwest Atlantic. Can Spec Pub Fish Aquat Sci 100

Buresch KC, Gerlach G, Hanlon RT (2006) Multiple stocks of longfin squid Loligo pealeii in the NW Atlantic: stocks segregate inshore in summer, but aggregate offshore in winter. Mar Ecol Prog Ser 310:263-270

Gerlach G, Buresch KC, Hanlon RT (2012) Population structure of the squid Doryteuthis pealeii on the eastern coast of the USA: Comment on Shaw et al. (2010). Mar Ecol Prog Ser 450:281-283

Knutsen H, Jorde PE, Andre C, Stenseth NC (2003) Finescaled geographical population structuring in a highly

Editorial responsibility: Christine Paetzold, Oldendorf/Luhe, Germany mobile marine species: the Atlantic cod. Mol Ecol 12: 385-394

Pritchard JK, Stephen M, Donnelly PJ (2000) Inference of population structure using multilocus genotype data. Genetics 155:945-959

Ruzzante DE, Taggart CT, Cook D (1998) A nuclear DNA basis for shelf- and bank-scale population structure in northwest Atlantic cod (Gadus morhua): Labrador to Georges Bank. Mol Ecol 7:1663-1680

Ryman N, Palm S (2006) POWSIM: a computer program for assessing statistical power when testing for genetic differentiation. Mol Ecol Notes 6:600-602

Shaw PW, Hendrickson L, McKeown NJ, Stonier T, Naud MJ, Sauer WHH (2010) Discrete spawning aggregations of loliginid squid do not represent genetically distinct populations. Mar Ecol Prog Ser 408:117-127

Submitted: February 3, 2012; Accepted: February 7, 2012

Proofs received from author(s): February 27, 2012 